\title{
L'énigme des contraintes grammaticales sur les sujets poyaudins
}

\author{
Benjamin Massot ${ }^{1}$ \\ Institut für Linguistik/Romanistik, U. Stuttgart, Keplerstr. 17, D-70174 Stuttgart, Allemagne
}

\begin{abstract}
Résumé. Notre contribution fait état de notre étude en cours des sujets du poyaudin, un patois d'oui. D'une part, en poyaudin, tous les syntagmes nominaux ne sont pas éligibles pour la position syntaxique de sujet : ils doivent pour cela être suffisamment définis/topicaux. Les autres syntagmes nominaux, s'ils doivent exprimer le sujet sémantique de la prédication, doivent être insérés dans des structures syntaxiques où ils peuvent apparaitre en position d'objet : comme élément introduit par y a dans une clivée, ou comme sujet réel d'un verbe à la tournure impersonnelle. D'autre part, les syntagmes nominaux sujets sont (très) fréquemment redoublés d'un clitique nominatif. Nous proposons alors un ensemble d'hypothèses : (i) Les contraintes qui pèsent sur les sujets dépendent crucialement du fait que les syntagmes candidats à la position syntaxique de sujet sont évalués sur une échelle de définitude/accessibilité/topicalité. (ii) Les clitiques nominatifs portent un trait d'accord de type défini/accessible/topical. Ce trait implique que le redoublement clitique du sujet n'est possible que lorsque le sujet est évalué sur l'échelle en question par une valeur compatible avec le trait du clitique nominatif. Ainsi, certains sujets, trop bas sur cette échelle, ne déclenchent pas l'accord par redoublement clitique. (iii) Les syntagmes nominaux trop bas sur l'échelle pour être éligibles en position de sujet trouvent une place adéquate dans les clivées et les tournures impersonnelles parce qu'ils s'y trouvent dans des positions syntaxiques d'objets, qui n'ont pas de restriction dans ce sens.
\end{abstract}

\begin{abstract}
The enigma of grammatical constraints on subjects in Poyaudin. Our paper summarizes our ongoing study of subjects in Poyaudin. First, in Poyaudin (an oïl dialect), not all noun phrases are possible syntactic subjects : they have to be topical/definite to a certain degree. When not sufficiently topical/definite, the semantic subject of the predication has to appear in a syntactic object position, either as the object of the presentational $y$ a in a cleft sentence or following a non-agreeing impersonal verb. Se-
\end{abstract}

1. benjamin.massot@wanadoo. fr 
cond, the (syntactic) subject noun phrases are (very) frequently doubled by a nominative clitic. Therefore, we propose a set of hypotheses : (i) The contraints applying to subjects basically depend on their evaluation on a scale of definiteness/accessibility/topicality. (ii) $\mathrm{N}$ ominative $\mathrm{c}$ litics b ear a definite/accessible/topical feature that h as to a gree $w$ ith the syntactic subject. If the subject is not sufficiently d efinite/accessible/topical, then it is not doubled by a nominative clitic. (iii) The appropriate position for noun phrases at the bottom of the scale is in cleft and impersonal sentences, since these non-subject positions are not restricted to the more definite/accessible/ topical noun phrases.

\section{Présentations}

\subsection{Les dialectes d'oui et le poyaudin en linguistique française}

Notre objet d'étude est le poyaudin, un patois français, c'est-à-dire un dialecte primaire de langue d'oui ${ }^{1}$. Le poyaudin est parlé, ou plutôt connu, par quelques derniers locuteurs, qui en général n'en gardent pas d'usage quotidien. La définition et la délimitation du poyaudin est somme toute assez simple, puisque le critère définitoire pratiqué est purement géographique : il s'agit du patois (dans le sens dialecte galloroman primaire) de la Puisaye, région naturelle du sud-ouest de l'Yonne (cantons de Saint-Fargeau, Toucy, Saint-Sauveur-en-Puisaye, Bléneau, Charny), du nord-ouest de la Nièvre (canton de Saint-Amand-en-Puisaye), et de quelques communes limitrophes du Loiret. On y associe souvent la région voisine de la Forterre (canton de Courson-les-Carrières). La figure 1 en annexe montre l'étendue de la Puisaye sur la carte de l'ALB, sur laquelle nous avons également reporté les points de l'ALF pertinents. La seule difficulté de délimitation qui peut apparaitre tient dans le soupçon d'un continuum entre poyaudin et français vernaculaire, d'autant plus envisageable que les deux idiomes sont très proches. Ainsi, sur le plan linguistique, le poyaudin, malgré sa présence dans la Bourgogne administrative, est à rapprocher des parlers du centre, notamment du Berrichon, plutôt que des parlers bourguignons-morvandiaux.

Notre espoir, en étudiant un dialecte d'oui, est celui d'Auger [1, p. 382] :

[N]ous espérons [...] que l'étude détaillée d'une langue qui n'est pas « tout à fait » le français permettra d'approcher de façon plus objective et d'éclairer une question particulièrement épineuse de la linguistique française.

En revanche, d'après notre expérience dans l'observation des dialectes d'oui [2, chap. 5-6, 3, 4], nous nous distancions de l'a priori suivant [1, p. 382] :

[I]l est raisonnable de penser que le picard représente, dans une large mesure, ce que serait devenu le français si le développement de ce dernier n'avait été constamment freiné par les instituteurs, les grammairiens et les académiciens[.]

En effet, nous avons souvent constaté que les dialectes d'oui peuvent être plus conservateurs que le français, et en particulier que le français vernaculaire n'est pas systématiquement «en retard » sur les dialectes d'oui. Le picard lui-même en offre un exemple net avec son emploi de ne très conservateur en comparaison avec le français vernaculaire [5]. Pour les clitiques sujets, qui vont nous intéresser dans cet article, King et Nadasdi [6] indiquent que ceux du français terre-neuvien se comportent comme ceux du français classique, et non comme ceux du français vernaculaire. 
Notre démarche est celle de l'étude de la microvariation syntaxique : la même question est posée à un ensemble de variétés proches, les dialectes d'oui à côté du français standard et du français vernaculaire. On espère alors obtenir une image plus fine des possibilités d'implémentation d'un point de grammaire donné. La présente étude souhaite donc élargir les travaux déjà faits sur le français dans ses différentes variétés et sur le picard, en présentant des faits du poyaudin. Les enquêtes en cours du projet SyMiLa (cf. ci-après) permettront d'élargir encore la palette des dialectes pris en compte.

\subsection{Thématique de l'étude}

La «question épineuse de la linguistique française » d'Auger [1, 7] est en gros celle que nous reprenons ici, puisque nous étudierons la morphosyntaxe des sujets en poyaudin. Comme il apparaitra par la suite, cette étude est en chantier, présentée ici dans l'état actuel de nos avancées. La limitation principale tient dans le fait que nous avons pour l'instant dépouillé une seule enquête fournissant les données à la base de notre étude. Non seulement nous pourrons ensuite ajouter les données produites par un autre locuteur du poyaudin, mais en plus nous pourrons élargir les données à des locuteurs d'autres régions couvertes par SyMiLa.

Dans le cadre de la linguistique du français, l'étude des sujets n'est pas un champ vierge, bien au contraire. Particulièrement pertinentes pour les patois sont l'étude et l'analyse des clitiques nominatifs et de la cooccurrence d'un sujet plein et d'un clitique nominatif. Cf., parmi beaucoup d'autres, Auger [1, 7, 8, 9], Culbertson [10], De Cat [11, 12], Poletto [13], Rizzi [14], Zanuttini [15] et Zribi-Hertz [16]. Inspirée de la littérature sur le français, le picard, et les dialectes nord-italiens, notre supposition est que l'on sera amené à se poser les questions suivantes :

1. Les clitiques nominatifs sont-ils des sujets au sens syntaxique, ou des affixes grammaticaux du verbe fléchi?

2. Les sujets pleins occupent-ils la position syntaxique de sujet, ou une autre position, disloquée?

3. Conséquence de 1 et 2 : La cooccurrence des sujets pleins et des clitiques nominatifs relève-t-elle d'une dislocation, ou d'un redoublement clitique?

4. Quels sont les sujets pleins autorisés, et où et comment les sujets pleins interdits sont-ils encodés syntaxiquement?

5. Quels sont les mécanismes qui peuvent être invoqués pour rendre compte des contraintes qui pèsent sur les sujets poyaudins?

\subsection{Corpus et méthodologie}

Le corpus sur lequel se base cette étude est l'ensemble des réponses fournies par un locuteur du poyaudin (ci-après $\mathrm{CR}$ ) aux stimulus de l'enquête SyMiLa.

Le projet SyMiLa (Syntactic Microvariation in the Romance Languages of France ${ }^{2}$ ), dans son volet «nouvelles enquêtes », a en effet développé un questionnaire syntaxique. La méthodologie de l'enquête est la suivante : on présente à un locuteur une série de quelques 350 phrases françaises (nommées ci-après stimulus, abréviation : st.) que l'on demande de traduire en patois. Les stimulus sont réunis en séquences ayant une cohérence narrative. Ainsi, les traductions (nommées ci-après réponses, abréviation : rép.) apparaissent presque toujours dans un contexte, que nous indiquerons lorsque ce sera pertinent. Le début de la séquence 2 peut servir d'illustration (tableau 1).

Le locuteur présenté ici, CR, a produit ses réponses directement après avoir simultanément lu et entendu chaque stimulus lu à haute voix par l'enquêteur. Nous avons parfois sug- 
Tableau 1. Extrait du questionnaire SyMiLa

\author{
Séquence 2 : Conte de l'âne peureux \\ $N^{\circ}$ phrase à faire traduire \\ 14 C'est l'histoire d'un âne qui n'avait presque jamais quitté son village. \\ 15 Il ne pouvait aller nulle part. \\ 16 Il était attaché à un piquet pour qu'il ne puisse pas courir dans le pré. \\ 17 C'était un âne gris, pas très grand, calme.
}

géré d'autres variantes, que CR a données comme réponses alternatives possibles lorsqu'il les a jugées grammaticales dans son parler. Il y a donc parfois plusieurs réponses à un même stimulus. Nous avons sélectionné les stimulus et les réponses que nous avons jugés pertinents pour l'étude de l'expression des sujets.

La transcription adoptée est basée sur la phonographie du français. Ses principes sont présentés à l'annexe A. Les exemples présentés sont donc tous produits par CR. Ils sont accompagnés du stimulus à l'origine de la réponse, et du numéro du stimulus dans le questionnaire. Lorsque le contexte précédent est pertinent pour l'analyse, nous l'indiquerons.

Nous étudions donc un idiolecte, en supposant que les données disponibles permettent une généralisation assez fiable sur la grammaire de CR. Les données dialectales étant ce qu'elles sont, c'est-à-dire diatopiquement très variables. même à petite échelle, il nous semble intéressant que notre étude puisse s'appliquer à l'échelle la plus basse de la diatopie, c'est-à-dire l'idiolecte. L'objectif, en élargissant par la suite l'ensemble des idiolectes pris en compte, n'est pas alors de s'approcher d'une grammaire commune (du poyaudin, ou du bourguignon, encore moins du français), mais de se donner une idée plus précise des grammaires individuelles possibles, et des contraintes et régularités qui se retrouvent d'un idiolecte à l'autre ${ }^{3}$.

\title{
2 Quels sujets en poyaudin ?
}

\subsection{Les clitiques nominatifs}

Les verbes fléchis sont accompagnés d'un clitique nominatif (1). Le paradigme de conjugaison est donné dans le tableau 2. Le fait que le clitique nominatif soit quasi obligatoire nous incitera à supposer qu'il a un statut morphologique. Dans la suite, nous verrons les quelques exemples où un verbe fléchi n'est pas accompagné d'un clitique nominatif, et nous y chercherons une explication. Seule la position proclitique est possible, aucun des stimulus de l'enquête SyMiLa avec inversion clitique n'a donné de réponse avec inversion. Elles ont été rejetées explicitement par les locuteurs lorsque nous en avons suggéré.

Sans surprise, les clitiques nominatifs sont séparables du verbe fléchi seulement par toutes sortes de pronoms objets proclitiques, comme 1 et $m$ en (2). (le clitique de négation ne est absent du poyaudin, il n'est donc pas pertinent ici.)

(1) il-avé pouẽ d kopẽ, i-vivé tou seul. (st. no 18 : N'ayant pas d'amis, il vivait seul.) 
Tableau 2. paradigme de conjugaison du poyaudin (verbes arivé et kôzé au présent)

\begin{tabular}{|c|c|c|c|}
\hline & pers. & arivé & kôzé \\
\hline \multirow{5}{*}{ sg. } & 1 & $j$-ariv- $\varnothing$ & (é)j-kôz- $\varnothing$ \\
\hline & 2 & $t$-ariv- $\varnothing$ & tu/té/te-kôz- $\varnothing$ \\
\hline & $3 \mathrm{~m}$ & $i l-\operatorname{ariv}-\varnothing$ & $i-k \hat{z} z-\varnothing$ \\
\hline & $3 f$ & al-ariv- $\varnothing$ & $a-k \hat{o} z-\varnothing$ \\
\hline & $3 n$ & sa $(1)-\operatorname{ariv}-\varnothing$ & sa-kôz- $\varnothing$ \\
\hline \multirow{4}{*}{ pl. } & 4 & $j$-ariv-õ (ou õn-ariv- $\varnothing$ ) & (é)j-kôz-õ (ou õ-kôz) \\
\hline & 5 & $V$-ariv-é & vou-kôzé \\
\hline & $6 \mathrm{~m}$ & il-ariv-o & $i-k \hat{z} z-\tilde{O}$ \\
\hline & $6 f$ & al/il-ariv-õ & a/i-kôz-õ \\
\hline
\end{tabular}

(2) j-I-é évu su mé sa-m-a sortu d 1 idé. (st. no 76-77 : Je l'ai eu su, mais je l'ai oublié.)

\subsection{Les syntagmes nominaux}

Concernant les syntagmes nominaux sujets (DP sujets), deux points sont à remarquer. Comme ils apparaissent accompagnés d'un clitique nominatif, on peut se demander s'ils sont dans la position syntaxique du sujet (SpecIP dans une certaine tradition générativiste), analyse solidaire d'une analyse des clitiques nominatifs comme affixes, ou bien s'ils sont disloqués (dans une position de topique de la périphérie gauche), analyse elle solidaire d'une analyse des clitiques nominatifs au niveau de la syntaxe. Comme la présence des clitiques est presque obligatoire (voir ci-dessous pour les exceptions), nous partons de l'hypothèse que les clitiques nominatifs sont des affixes, et que, si aucun autre indice ne vient le contredire, les DP sujets sont dans la position syntaxique du sujet (3). Les dislocations ne sont pas exclues, seulement, on n'en supposera justement que si un indice nous y pousse. En (4), on a une dislocation à gauche, comme l'indique la présence du pronom fort él. En (5), c'est une dislocation à droite. En (6), c'est la présence du pronom interrogatif koué qui indique la présence d'une dislocation à gauche. Le fait que léz ôt apparaisse à gauche du complémenteur (koué $k$ ) indique que pi léz ôt est peut-être à analyser comme syntaxiquement indépendant de la question. Enfin, les pronoms forts sujets se comportent comme les DP sujets (7).

(3) I mõd il-é idyo. (st. no 339 : Les gens sont idiots.)

(4) sa fam, él, al-aré bẽ voulu résté mé al-a pà pu.

(st. no 235 : Sa femme, elle, elle aurait bien voulu resté, mais elle n'a pas pu.)

(5) i-chãt ptét bẽ, s mérl! (st. n 208 : Comme ce merle chante bien!)

(6) pi léz ôt, koué $k$ il-ã-pãsõ ? (st. no 74 : Qu'en pensent les autres ?)

(7) bẽ moué $\boldsymbol{j}$-sé pà si $\boldsymbol{j}$-và dormi la. (st. no 143 : Moi, je ne sais pas si je vais dormir là.)

L'exemple (8) montre par ailleurs que le redoublement clitique du sujet est possible dans les subordonnées comme dans les principales. 
(8) é kãt la gamin al-é revnu 1-lãnmẽ, é bẽ I àn il-été pu dã sõ pré.

(st. $\mathrm{n}^{\circ} 70$ : Mais quand la fillette revint le lendemain, l'âne n'était plus dans son pré.)

\subsection{Les sujets Qu-}

Les pronoms interrogatifs sujets sont qi (noter la palatalisation) pour les humains (9), et koué pour les inanimés (10). Le déterminant interrogatif est qeul (11). Le pronom interrogatif élqeul est aussi possible (12). Ajoutons pour comprendre les exemples suivants que les questions Qu- présentées respectent le schéma suivant (qui n'est pas le seul en poyaudin, mais qui semble le plus caractéristique, et qui est revenu assez systématiquement dans les réponses) : mouvement $\mathrm{Qu}-$ + particule dõ (optionnelle) + complémenteur $k$ ou ki. La répartition de $k$ et ki n'est pas claire : (11b) montre par exemple que ki apparait aussi devant voyelle. Par ailleurs, (11a) montre que le complémenteur peut même ne pas être présent, ce que nous attribuons ici à la présence de la relative $k$ aré deû sou $d$ jujot.

(9) a. qi dõ $\mathbf{k}$ é déja la ? (st. no 115 : Qui est déjà arrivé ?)

b. si jamé ẽ jour tu-t-ã-và, qi dõ ki va alé ak toué?

(st. n ${ }^{\circ} 66$ : Mais si tu décides de partir, qui penses-tu qui t'accompagnera ?)

c. bõ bẽ qi ki fé koué isi ? (st. no 126 : Bon, qui fait quoi, isi ?)

d. bõ qi dõ ki fé koué isi ? (id.)

(10) koua dõ $\boldsymbol{k}$ é tõbé ? (st. no 161 : Qu'est-ce qui est tombé ?)

(11) a. qeul dõ pérson $k$ aré deû sou d jujot a-vouré alé viv la-bà ?

(st. no 104 : Quelle personne sensée accepterait d'aller y vivre ?)

b. qeul dõ vouazin ki ira chérché mé nveu, dã s kà-la?

(st. no 136 : Quelle voisine est-ce qui ira chercher mes neveux, alors ?)

(12) élqeul dõ d vou ki doua parti d bon eur?

(st. $\mathrm{n}^{\circ} 145$ : Lequel d'entre vous doit partir de bonne heure ?)

L'analyse possible de qui comme complémenteur en français (en distribution complémentaire avec que en tête de relative) date d'au moins Moreau [17]. Cette analyse s'oppose à l'analyse de la paire qui/que comme pronoms relatifs/interrogatifs selon le critère de la fonction (sujet vs. COD). Les faits poyaudins confirment que l'abandon de l'analyse comme pronoms ne doit pas être craint, puisque le détail montre que l'interrogatif qui a subi une évolution que le relatif/complémenteur qui n'a pas vécue, à savoir la palatalisation de / $\mathrm{k} / \mathrm{en} / \mathrm{c} /$ devant /i/. La paire minimale poyaudine ki/qi (9bcd) montre que ces deux items sont traités indépendamment dans la grammaire. Auger [7, pp. 11-12] utilise également la palatalisation (et son absence) pour aider à identifier la nature de $/ \mathrm{ki} /$ en tête de relative sujet en picard, même si les faits picards ne sont pas parfaitement calquables sur nos faits poyaudins.

La conséquence de cette analyse de $k / k i$ comme complémenteur, c'est que les verbes des interrogatives partielles sujets ne sont pas en général accompagnés d'un clitique nominatif. (11a) montre que lorsque le DP QU- n'est pas suivi du complémenteur, le clitique nominatif réapparait. Nous poserons la question, dans la section 3, de ce qui peut motiver les deux cas observés qui fonctionnent comme (11a). Qu'est-ce qui peut être différent d'avec le cas (11b) (également 2 exemples dans le corpus)?

\subsection{Dans les relatives sujets}

Les relatives sujets de CR sont introduites par le complémenteur $\mathrm{k} / \mathrm{ki}$ (cf. argumentation cidessus), et le verbe de la relative n'est pas accompagné d'un clitique nominatif. 
(13) a. $\quad s$-é 1 istouèr $d$ un àn $\mathbf{k} \varnothing$ été kazimã jamé parti $d$ ché lui $+d$ sõ vilaj. (st. no 14 : C'est l'histoire d'un âne qui n'avait presque jamais quitté son village.)

b. la gamin, $\boldsymbol{k i} \varnothing \mathrm{s}$-lêsé pà dékourajé, a-s-é pri a yi-kozé (st. $n^{\circ} 26$ : La fillette, que rien ne décourageait, lui adressa la parole.)

\subsection{Absences de clitique nominatif}

Nous avons annoncé que nous analysons les clitiques nominatifs comme des affixes, marquant sur le verbe un accord avec le sujet (lequel est exprimé par ailleurs ou pas). Dans quelques cas, il n'apparait aucun clitique nominatif, ce qui semble poser certains problème à notre hypothèse, à en croire la littérature. Bien que nous ne le croyions pas (cf. discussion en 3.2), il faudra néanmoins rendre compte de ces cas. Certains semblent assez triviaux, d'autres sont moins attendus.

Notons tout d'abord que le clitique nominatif impersonnel est présent dans les verbes météorologiques, sous la forme i(l) ou sa (14).

(14) a. oui $\mathbf{i}$-pleu for $\mathbf{i}$-pleu vrêmã oui pi $\mathbf{i}$-pleuvra davãtaj demẽ (st. $n^{\circ} 174$ : Il pleut fort, Il pleut vraiment. Il pleuvra davantage demain.)

b. Sa-pleu for sa-pleu vrêmã pi sa-pleuvra davãtaj demẽ (st. no 174 : id.)

Ensuite, faloué comme $y$-a apparaissent en général sans clitique nominatif (15).

(15) a. mé $\varnothing$ y-a riẽ a yeu mõtré (st. no 205 : mais il n'y a rien à leur montrer)

b. $\varnothing$ fo vrêmã pà ét normal pou fề dê afê paréy

(st. $\mathrm{n}^{\circ} 252$ : Il faut vraiment ne pas être normal/normaux pour faire des choses pareilles.)

Les tournures impersonnelles, comme en (16), ne prennent pas de clitique nominatif.

(16) $\varnothing$ sãblé $k$ nô ôt vouézẽ il-avẽ désidé d parti eu osi

(st. $\mathrm{n}^{\circ} 237$ : Il semblait que nos autres voisins avaient décidé de partir, eux aussi.)

Enfin, les clitiques nominatifs sont, sans surprise, absent des impératifs (17).

(17) nõ $\varnothing$ va-zi, $\varnothing$ ouvér-la (st. $n^{\circ} 168$ : Non, vas-y, ouvre-la!)

Il reste un cas, celui des exemples, rares mais pas exclus, où un DP sujet n'est pas redoublé d'un clitique nominatif. Là-aussi notre étude est loin d'en être à un stade satisfaisant, et notre impression est bien que ces cas ne seront pas négligeables. Pour la difficulté qu'ils représentent dans notre analyse, cf. §3.

(18) de qeul dõ kouleur ke lê volê sõ pẽ ?

(st. $\mathrm{n}^{\circ} 215$ : De quelle couleur sont donc peints les volets?)

(19) a. vou-ll-avé ti vu 1 film ke y-a tou l+ ke tou I mõn parl?

(st. $\mathrm{n}^{\circ} 267$ : Et ce film dont tout le monde parle, l'avez-vous vu ?)

b. 1 film ke tou I mõd ã-parl vou-1l-avé ti vu ? (st. n 267 : id.)

c. *... ke tou 1 mõd i-parl

Les relatives telles qu'exposées en (13ab) manquent également d'un clitique nominatif, tout comme presque toutes les interrogatives partielles en 2.3. Nous essaierons d'en rendre compte également.

\subsection{Contraintes observées sur les DP sujets}

En ce qui concerne les DP sujets, ils sont limités à un sous-ensemble de DP. Les exemples suivants montreront que certains DP sujets dans le stimulus de l'enquête SyMiLa n'ont pas 
été traduits par un sujet en poyaudin. Les résultats sont systématiques au cours de l'enquête, les locuteurs n'ont pas accepté les versions proposées avec le DP en position syntaxique de sujet (avec ou sans redoublement clitique).

(20) a. ẽ jour, y-a un gamin ki s-é rtrouvé ã fas de lui. (st. $n^{\circ} 22$ : Un jour, une fillette s'est retrouvée en face de lui)

b. * ẽ jour un gamin (a-)s-é rtrouvé ã fas de lui.

(21) a. $\quad y$-a apsolumã pérson $k$ é eureu si il-a pouẽ $d$ libérté. (st. no 51 : Absolument personne n'est heureux sans aucune liberté.)

b. *apsolumã pérson (il-)é eureu si il-a pouẽ d libérté

(22) a. j-é ajté ẽ peu pu d pẽ, dê foué $k$ i-vién qeukẽ $\boldsymbol{d}$ ôt.

(st. no 342 : J'ai acheté un peu plus de pain au cas où quelqu'un d'autre viendrait.)

b. *j-é ajté ẽ peu pu d pẽ, dê foué $k$ qeukẽ d ôt (i-)vién.

Quid alors des candidats malheureux ? Tout d'abord, on remarque que un gamin, absolument pérson et qeukẽ $d$ ôt sont des DP indéfinis. La remarque à faire est double :

1. Les DP indéfinis ne sont pas redoublables par un clitique nominatif.

2. Les DP indéfinis ne sont pas non plus non-redoublés, mais apparaissent dans une autre structure syntaxique, dans laquelle ils n'occupent pas la position syntaxique de sujet.

La solution la plus fréquente pour que le DP n'apparaisse pas en position de sujet, qui est d'ailleurs une construction très favorisée en français vernaculaire, c'est la construction clivée par $y$-a comme en (20) et (21) (19 cas sur les 22 possibles dans le corpus, cf. tableau 5, annexe C). La solution en (22) est moins fréquente (3 cas sur 22). Le DP indéfini y apparait en position de sujet réel du verbe vién à la forme impersonnelle.

Enfin, une observation semble pertinente pour cerner ce qui semble être en jeu ici. Dans 4 cas, CR a traduit un sujet défini (la porte), encodé comme sujet syntaxique dans le stimulus, par une clivée en $y$-a, comme en (23).

(23) regard dõ st ôt mézõ. $y$-a la port $k$ é ouvért.

(st. $\mathrm{n}^{\mathrm{o}} 213-214$ : Regarde cette autre maison. La porte en est ouverte.)

Dans le contexte indiqué, la porte n'est pas le topique actuel, puisque le stimulus 213 établit cette autre maison dans le rôle de topique. La réponse de CR réorganise la phrase pour que le DP non-topique la port n'apparaissent pas en position syntaxique de sujet, en le clivant par $y-a$.

\section{Propositions d'analyse}

Pour résumer, les faits dont nous essayons de rendre compte sont les suivants :

- Les DP sujets sont presque systématiquement redoublés par un clitique nominatif.

- Quelques verbes fléchis se présentent sans clitique nominatif ni DP sujet.

— Seuls les DP définis sont éligibles à la position syntaxique de sujet.

- Les DP indéfinis, non éligibles à la position syntaxique mais candidats au rôle sémantique de sujet du prédicat, sont encodés soit comme élément clivé par le présentatif $y$-a, soit comme sujet réel d'un verbe à la tournure impersonnelle.

- Les relatives sujets sont dépourvues de clitique nominatif.

- Les interrogatives sujets sont parfois dépourvues de clitique nominatif, parfois accompagnées d'un tel clitique. 


\subsection{Hypothèses}

À ce point de notre étude, nous proposons une analyse faisant les hypothèses suivantes. Les hypothèses $1,2 \mathrm{~b}$ et 3 constituent le cœur de notre analyse.

1. Les DP concernés se placent sur une échelle de définitude/accessibilité/topicalité, qu'il est encore nécessaire d'identifier exactement. Ils se répartissent en trois catégories :

A : les DP en haut de l'échelle, tels la gamin en (2);

B : les DP au milieu de l'échelle, tel tou 1 mõd en (19) et lê volê en (18);

C : les DP en bas de l'échelle, tel pérson ou qeukẽ $d$ ôt en (21) et (22). D'après la littérature (cf. [10, pp. 119-122] et les travaux qu'elle cite), les « variables QU-» (DP interrogatifs et pronoms relatifs) sont également en bas de l'échelle, ce qui correspond presque exactement à nos données.

2. Les sujets syntaxiques et le verbe s'accordent selon deux marques :

a) la terminaison verbale porte des traits de personne et de nombre;

b) le clitique nominatif porte, en plus des traits de cas (nominatif), de personne (variable) et de nombre (variable), un trait de définitude/accessibilité/topicalité (marqué comme défini/accessible/topical à au moins un certain point).

3. Les DP qui apparaissent en position syntaxique de sujet doivent respecter la contrainte ${ }^{4}$ suivante : leur référent doit être défini/accessible/topical à un certain degré.

4. Les syntagmes candidats à la position syntaxique de sujet qui ne respectent pas la contrainte énoncée en 3 sont inéligibles pour cette position et doivent être encodés en position d'objet. La construction clivée en $y$-a est une telle possibilité, la tournure impersonnelle en est une autre.

Les constructions clivées se prêtent bien au rattrapage des candidats malheureux, puisqu'elles permettent d'exprimer des prédications dans lesquelles le sujet de la prédication ne correspond pas nécessairement au topique de la proposition (cf. par exemple [18]).

5. L'hypothèse $2 b$ demande à être précisée. Puisque deux exemples ne montrent pas de redoublement clitique, il faut accepter que cette règle d'accord est partielle, dans le sens ou l'accord n'est pas toujours exigé/possible. C'est ce que Culbertson [10] nomme «feature mismatch ».

L'absence de clitique dans les cas strictement impersonnels peut alors trouver une explication simple : le clitique nominatif exige un sujet référentiel, sans quoi sa présence crée un décalage de traits dans l'opération d'accord qui rend l'énoncé agrammatical. L'opposition entre verbes météorologiques et véritables impersonnels ( $y$-a et faloué) peut se comprendre si l'on admet l'hypothèse que les verbes météorologiques sont «moins impersonnels » que faloué et $y$-a [19].

6. Nous avons alors à faire à deux contraintes distinctes qui opèrent sur un critère présent sur la même échelle, et qui aboutissent à trois comportements. D'une part, les DP sujets A exigent du verbe qu'il marque un accord de définitude/accessibilité/topicalité. D'autre part, les DP sujets B ne déclenchent pas l'accord par redoublement clitique : l'hypothèse $2 \mathrm{~b}$ rend compte de la limite entre $\mathrm{A}$ et $\mathrm{B}+\mathrm{C}$. Enfin, les DP de type $\mathrm{C}$, qui pourraient simplement ne pas déclencher de redoublement clitique, sont rejetés par la contrainte 3 qui les rend non-éligibles à la position de sujet, avec les conséquences que l'on a vues : l'hypothèse 3 rend compte de la limite entre $A+B$ et $C$. 


\subsection{Discussion}

Un certain nombre de points se prêtent à discussion et remarques.

(i) Le fait de poser deux limites (en particulier à cause des cas B) est soutenu par ailleurs par l'observation que certains idiomes ne connaissent qu'une des deux contraintes : on répartit souvent les dialectes nord-italiens selon le degré d'avancement des sujets qui déclenchent le redoublement clitique (hypothèse de type $2 b$ ). Mais rien ne semble indiquer que les DP qui ne déclenchent pas ce redoublement ne soient pas possibles en position de sujet [14].

L'anglais, l'allemand, l'espagnol, l'italien (standard), pourraient être citées comme langues connaissant au contraire seulement la contrainte 3 , cf. point (v) ci-dessous.

(ii) Les deux limites indiquent que la contrainte $2 \mathrm{~b}$ et la contrainte 3 sont régies par un trait de définitude/accessibilité/topicalité réglé différemment sur l'échelle considérée.

(iii) Les constructions clivées ne sont pas réservées aux candidats malheureux, simplement, elles sont un asile idéal.

(iv) S'il se confirmait que pour lê volê de (18), leur trait non-animé est pertinent, il faudrait envisager qu'une échelle d'animacité (ou tout du moins une échelle la prenant en compte) soit pertinente pour la contrainte $2 b$.

(v) La contrainte 3, ou une version moins sévère (non pas une interdiction, mais l'assignation d'une marque) est connue dans d'autres langues, avec d'autres effets (à rapprocher de certaines explications de [20] sur les différences de traitement des sujets nontopicaux dans divers langues). Les candidats $\mathrm{C}$ reçoivent un accent focal en anglais, et en allemand. En allemand, ils peuvent ne pas recevoir d'accent focal s'ils cèdent leur place privilégiée dans le Vorfeld (la position pré-verbale dans les propositions à verbe second) à un autre membre de la proposition. En espagnol et en italien, on trouve des sujets post-verbaux.

(vi) Dans le cas de relatives, soit $\mathrm{k} / \mathrm{ki}$ est analysé comme un pronom, qui doit donc porter des traits de nombre, genre, personne, et définitude/topicalité/accessibilité, et l'absence d'accord par clitique nominatif sur le verbe laisse supposer que le pronom est faiblement défini/topical/accessible. Soit, comme nous le provilégions, $k / k i$ est un simple complémenteur, et il ne possède donc pas de trait de définitude avec lequel un clitique nominatif puisse marquer l'accord. En un sens, ce cas se rapprocherait alors de celui des impersonnels stricts (cf. hypothèse 5 ci-dessus). On peut aussi supposer qu'une simple trace du sujet est présente dans la relative, mais que cette trace ne possède pas les traits compatibles avec le clitique nominatif, empêchant le redoublement clitique du sujet.

(vii) Dans le cas des interrogatives, la même analyse peut être proposée que pour les relatives, en supposant que la structure et les contraintes sont identiques pour la partie de la phrase sous la portée du complémenteur $\mathrm{k} / \mathrm{ki}$.

(viii) L'observation des deux cas du type (11a), où le DP QU- n'est pas suivi du complémenteur et où le redoublement clitique du sujet a lieu, pose les deux problèmes suivants. Quelle est la structure exacte de la phrase et en quoi est-elle différente de celle avec complémenteur de (11b) ? Et, comment justifier que le sujet qeul dõ pérson $k$ aré deu sou d jujot soit suffisamment haut sur l'échelle régissant le redoublement clitique des sujets chez CR, alors même que les DP QU- sont réputés en bas de l'échelle, et alors même que rien ne semble lui conférer de définitude/topicalité/accessibilité particulière? 


\section{4 À suivre...}

Il nous reste à souhaiter poursuivre cette étude en poyaudin même, et l'étendre à plus de parlers d'oui, en particulier en nous appuyant sur les enquêtes [21] ailleurs qu'en Puisaye. Nous aimerions ainsi explorer les questions suivantes.

(a) Le traitement des sujets, même lorsqu'il est différent du poyaudin de CR, reflète-t-il l'échelle qui semble être en vigueur en poyaudin ? En particulier, si différents types de sujets sont traités différemment, est-ce que l'échelle (d'implication) proposée ici est respectée?

(b) Nous savons déjà que d'autres enquêtes pas encore dépouillées révèleront que certains locuteurs semblent partager la contrainte 3, mais qu'elle est seulement optionnelle. Quelles peuvent être les conséquences théoriques d'une telle optionalité ?

(c) Quel type d'échelle semble le plus adéquat : une échelle basée sur le type de référence (définitude) ou sur une dimension de structure informationnelle (topicalité ou accessibilité) ?

(d) Est-ce que les deux contraintes $2 \mathrm{~b}$ et 3 agissent vraiment sur la même échelle, ou bien est-ce qu'il faudra considérer deux échelles distinctes?

(e) Le traitement des sujets peut-il être corrélé à d'autres traitements ? Par exemple, au traitement de ne, ou de certains aspects de la conjugaison (forme du clitique et terminaison de la personne 4 , terminaison de la personne $6, \ldots$ ).

\section{Notes}

\footnotetext{
${ }^{1}$ Nous notons < oui > plutôt que < oïl > pour inviter le lecteur à lire [wi] plutôt que [oj(1)] ou [oil]. Cf. la courte discussion dans Martin [22].

${ }^{2}$ http://blogs.univ-tlse2.fr/symila/

${ }^{3}$ La méthode idiolectale est exposée dans Barra Jover [23] et exploitée, en dehors de nos propres publications, notamment par Iglesias et Larrivée [24].

${ }^{4}$ Notre emploi du terme contrainte n'implique pas que nous placions notre analyse dans le cadre de la théorie de l'optimalité. De même que le terme de construction (clivée) dans la suite n'implique pas que nous adoptions la grammaire de construction comme cadre d'analyse.
}

\section{Références}

[1] J. Auger. «Le redoublement des sujets en picard ». French Language Studies 13 (2003), pp. 381 404.

[2] B. Massot. «Français et Diglossie. Décrire la situation linguistique française contemporaine comme une diglossie : arguments morphosyntaxiques ». Thèse de doctorat. Saint-Denis : Université Paris 8 (2008). URL : http: //tel.archives-ouvertes.fr/tel-00726999.

[3] B. Massot. «Deux faits de morphologie du nombre dans le domaine nominal en poyaudin (et un peu en français)». In : Études de linguistique gallo-romane. Sous la dir. de M. Barra Jover et al. Sciences du Langage. Saint-Denis : PUV (2012), pp. 279-294.

[4] B. Massot. «Aspects de la morphosyntaxe des sujets en poyaudin ». In : Actes du colloque SyMiLa 2015. U. Toulouse 2 J. Jaurès. (). En prép.

[5] J. Auger et A.-J. Villeneuve. «Ne deletion in Picard and in regional French : Evidence for distinct grammars ». In : Social Lives in Language - Sociolinguistics and multilingual speech communities. Sous la dir. de M. Meyerhoff et N. Nagy. Amsterdam : Benjamins (2008), pp. 223 247.

[6] R. King et T. Nadasdi. «Left dislocation, number marking and (non-)standard French ». Probus 9 (1997), pp. 267-284. 
[7] J. Auger. «Les pronoms clitiques sujets en picard : une analyse au confluent de la phonologie, de la morphologie et de la syntaxe». French Language Studies 13 (2003), pp. 1-22.

[8] J. Auger. «Pronominal Clitics in Québec Colloquial French : A Morphological Analysis». Thèse de doct. Philadelphia: University of Pennsylvania (1994). URL : http: / / repos i tory . upenn.edu/ircs_reports/174.

[9] J. Auger. «Les clitiques pronominaux en français parlé informel : une approche morphologique ». Revue québécoise de linguistique 24.1 (1995), pp. 21-60.

[10] J. Culbertson. «Convergent evidence for categorial change in French : from subject clitic to agreement marker». Language 86 (2010), pp. 85-132.

[11] C. De Cat. «French subject clitics are not agreement markers ». Lingua 115 (2005), pp. 11951219.

[12] C. De Cat. «French dislocation without movement». Natural Language and Linguistic Theory 25.3 (2007), pp. 485-534.

[13] C. Poletto. «Subject clitic/verb inversion in north eastern Italian dialects ». University of Venice Working Parpers in Linguistics 3.1 (1993), pp. 95-137.

[14] L. Rizzi. «On the Status of Subject Clitics in Romance». In : Studies in Romance Linguistics. Sous la dir. d'O. Jaeggli et C. Silva-Corvalán. Dordrecht : Foris (1986), pp. 391-419.

[15] R. Zanuttini. Negation and clausal structure : a comparative study of Romance languages. Oxford studies in comparative syntax. New York; Oxford : Oxford University Press (1997).

[16] A. Zribi-Hertz. «The Syntax of Nominative Clitics in Standard and Advanced French». In : Paths Towards Universal Grammar : Studies in Honor of Richard S. Kayne. Sous la dir. de G. Cinque et al. Washington, D.C. : Georgetown University Press (1994), pp. 453-472.

[17] M.-L. Moreau. «L'home que je vois qui est venu. Qui, que : relatifs et conjonctions ». Langue Française 11 (1971) : Syntaxe transformationnelle du français, pp. 77-90.

[18] K. Lambrecht. «Presentational cleft constructions in spoken French ». In : Clause combining in grammar and discourse. Sous la dir. de J. Haiman et S. Thompson. Amsterdam : John Benjamins (1988), pp. 135-179.

[19] M.-O. Hinzelin et G. Kaiser. « Le paramètre du sujet nul dans les variétés dialectales de l'occitan et du francoprovençal ». In : Études de linguistique gallo-romane. Sous la dir. de M. Barra Jover et al. Sciences du Langage. Saint-Denis : Presses Universitaires de Vincennes (2012), pp. 247 260.

[20] K. Lambrecht. Information Structure and Sentence Form. Cambridge : Cambridge university press (1994).

[21] Projet SyMiLa. Syntactic Microvariation of the Romance Languages of France. URL : http: //blogs.univ-tlse2.fr/symila/.

[22] E. Martin. Le Courrier de Vaugelas 24 (1875). 15 mars 1875, p. 187. URL: www. gallica. fr.

[23] M. Barra Jover. Propriedades léxicas y evolución sintáctica. El desarollo de los mecanismos de subordinación en español. La Coruña : Toxosoutos (2002).

[24] O. Iglesias et P. Larrivée. «Une approche idiolectale de la chute de « ne » en français contemporain ». SHS Web of Conferences 8 (2014), pp. 2397-2411. DOI : 10 . 1051 / shsconf / 20140801179. URL: http://dx.doi.org/10.1051/shsconf/20140801179.

[25] G. Taverdet, éd. Atlas linguistique et ethnographie de la Bourgogne. Paris : CNRS (1984).

\section{A Système de transcription}

Nous recyclons la phonographie du français, en fixant la valeur phonographique de base (relation phonographique bi-univoque : $\langle\mathrm{g}>$ note toujours $/ \mathrm{g} /, \mathrm{s} /$ est toujours noté par $<\mathrm{s}\rangle$, $/ \mathrm{z} /$ par $\langle\mathrm{z}\rangle$, etc.), en supprimant tout graphème muet, en utilisant le tilde pour les voyelles 
nasales, l'accent circonflexe pour la longueur vocalique distinctive, en ajoutant le $<\mathrm{q}>$ et le digraphe $<\mathrm{gy}>$ pour les occlusives palatales $/ \mathrm{c} /$ et $/ \mathrm{f} /$, en séparant les mots comme en français, sauf pour l'utilisation systématique du tiret simple pour les clitiques pronominaux. L'apostrophe n'est pas utilisée. Les voyelles moyennes ne distinguent deux degrés d'aperture que pour /o/ vs. /o/, transcrites par un accent aigu et grave respectivement. /a/ est rendue par un accent grave. Nous renonçons aux distinctions d'aperture pour les voyelles longues (probablement neutralisées), et de longueur pour les voyelles nasales (également non-pertinente), ce qui évite les graphèmes à double diacritique. Le tableau 3 reprend l'ensemble des graphèmes retenus.

La prosodie est rendue par la ponctuation classique. Les ruptures syntaxiques et hésitations sont indiquées par le signe $<+>$.

Tableau 3. Les graphèmes retenus pour la transcription du poyaudin

\begin{tabular}{|c|c|c|c|c|c|c|}
\hline \multicolumn{4}{|c|}{ consonnes } & \multicolumn{3}{|c|}{ voyelles } \\
\hline $\begin{array}{l}/ \mathrm{p} /<\mathrm{p}> \\
/ \mathrm{b} /<\mathrm{b}> \\
/ \mathrm{f} /<\mathrm{f}> \\
/ \mathrm{v} /<\mathrm{v}> \\
/ \mathrm{m} /<\mathrm{m}> \\
/ \mathrm{r} /<\mathrm{r}>\end{array}$ & $\begin{array}{l}/ \mathrm{t} /<\mathrm{t}> \\
/ \mathrm{d} /<\mathrm{d}> \\
/ \mathrm{s} /<\mathrm{s}> \\
/ \mathrm{z} /<\mathrm{z}> \\
/ \mathrm{n} /<\mathrm{n}> \\
/ \mathrm{l} /<\mathrm{l}>\end{array}$ & $\begin{array}{l}/ \mathrm{c} /<\mathrm{q}> \\
/ \mathrm{f} /<\mathrm{gy}> \\
/ \mathrm{f} /<\mathrm{ch}> \\
/ 3 /<\mathrm{j}> \\
/ \mathrm{j} /<\mathrm{gn}> \\
/ \mathrm{j} /<\mathrm{y}>\end{array}$ & $\begin{array}{l}/ \mathrm{k} /<\mathrm{k}> \\
/ \mathrm{g} /<\mathrm{g}>\end{array}$ & $\begin{array}{l}\mid \mathrm{i} /<\mathrm{i}> \\
/ \mathrm{E} /<\mathrm{e}> \\
\\
\mid \mathrm{a} /<\mathrm{a}> \\
\mid \tilde{\varepsilon} /<\tilde{\mathrm{e}}> \\
\text { longueur } \mathrm{v}\end{array}$ & $\begin{array}{l}\mid \mathrm{y} /<\mathrm{u}> \\
\mid \emptyset /<\mathrm{eu}> \\
/ \mathrm{\partial} /<\mathrm{e}> \\
/ \tilde{\mathrm{a}} /<\tilde{\mathrm{a}}> \\
\text { ocalique : }\end{array}$ & $\begin{array}{l}/ \mathrm{u} /<\mathrm{ou}> \\
/ \mathrm{O} /<\mathrm{o}(\mathrm{o} \text { ò })> \\
/ \mathrm{a} /<\mathrm{a}> \\
/ \tilde{\mathrm{o}} /<\tilde{\mathrm{o}}> \\
/ \mathrm{V} / \quad<\hat{\mathrm{V}}>\end{array}$ \\
\hline
\end{tabular}

\section{B Situation géographique de la Puisaye}

La figure 1 montre l'emplacement géographique de la Puisaye sur la carte de l'atlas linguistique et ethnographique de la Bourgogne [25]. Nous y avons également reporté les points d'enquête de l'ALF présents sur ce territoire.

\section{Détails des sujets produits}

Les tableaux 4-5-6 détaillent le corpus concernant les réponses de CR qui traduisent un sujet plein du stimulus. La première colonne donne le type de DP (défini, pronom tonique, générique, indéfini, pronom relatif, mot QU-, DP QU-), la seconde colonne donne la réalisation syntaxique du stimulus, la troisième la réalisation syntaxique dans la réponse, la quatrième le nombre de cas relevés dans l'ensemble des réponses de CR, et la cinquième le numéro de l'exemple illustrant le cas en question. Rappelons que les nombres présents ne peuvent pas être pris absolument, puisqu'ils sont directement tributaires des choix faits par les auteurs du questionnaire. Les seules quantifications pertinentes sont celles comparant la fréquence relative de deux types de réponses possibles à un même type de stimulus.

Les principales abréviations sont les suivantes. SV : Sujet+Verbe (sans clitique nominatif); VS : Verbe+Sujet (inversion, sans clitique nominatif); RS : redoublement clitique du sujet; DG : dislocation à gauche du sujet; DD : dislocation à droite du sujet. Les exemples proposés devraient par ailleurs permettre de comprendre facilement les autres abréviations.

(24) vou-11-avé ti vu, 1 film ke y-a tou $1+$ ke tou 1 mõn parl? (st. $\mathrm{n}^{\circ} 267$ : Et ce film dont tout le monde parle, l'avez vous vu ?)

(25) la chmi ke ch-chui ã trẽ de rpâsé s-é la seul k tu-m-à ofért.

(st. $n^{\circ} 278$ : La chemise que je suis en train de repasser est celle que tu m'as offerte.) 


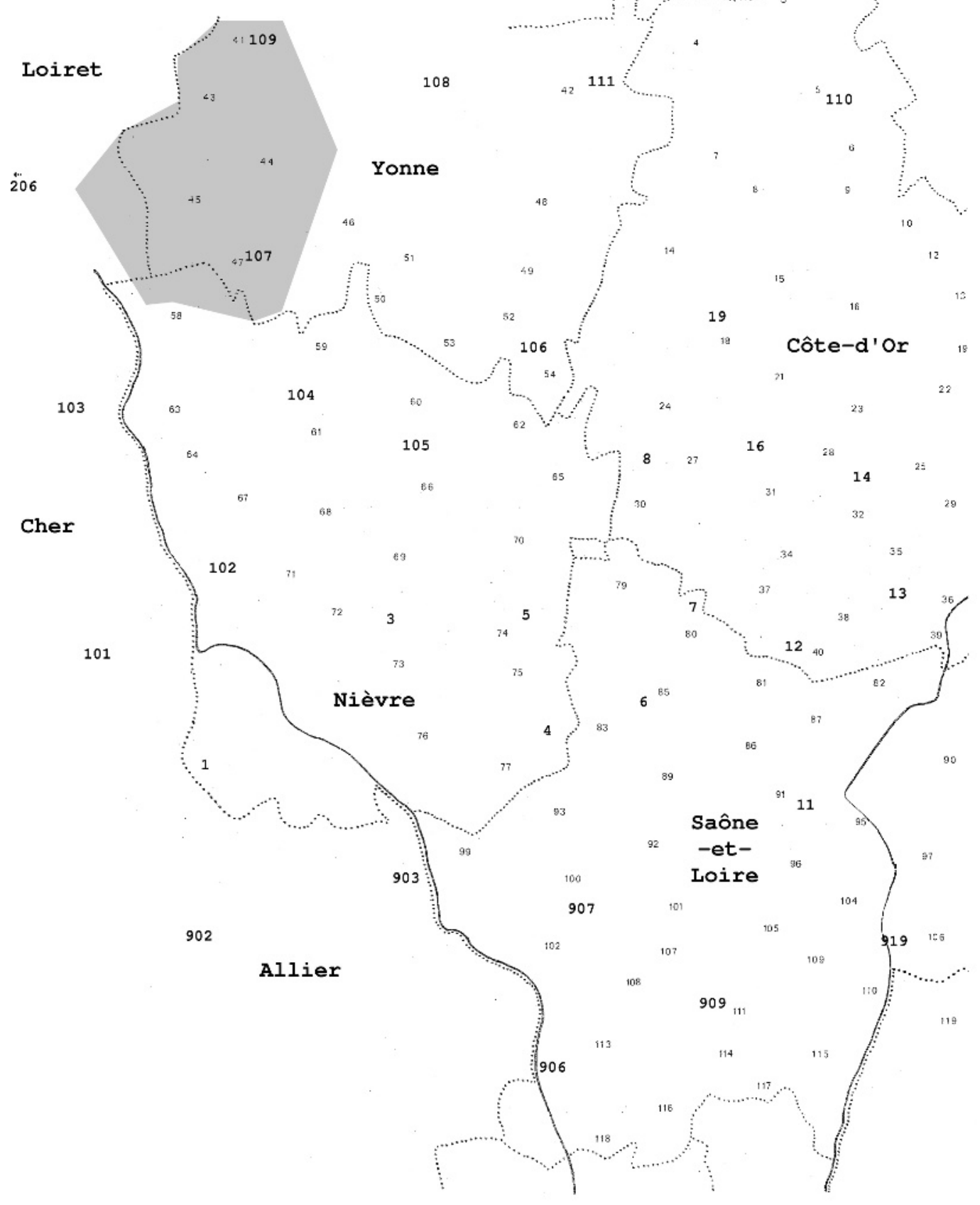

Fig. 1. La Puisaye sur l'ALB 
Tableau 4. Les sujets définis de CR

\begin{tabular}{|c|c|c|c|c|}
\hline S du st. & réal. ds st. & réal. ds rép. & $\mathrm{nb}$ & ex. \\
\hline \multirow[t]{22}{*}{ DP déf. } & \multirow[t]{17}{*}{ SV } & SV (ds rel.) & 1 & (24) \\
\hline & & RS (s-é) & 1 & $(25)$ \\
\hline & & RS (il) dans principale & 23 & $(26)$ \\
\hline & & RS (il) dans qu. totale & 6 & (27) \\
\hline & & RS dans qu. partielle. & 8 & $(28)$ \\
\hline & & RS ds sub. & 18 & $(26)$ \\
\hline & & total $R S$ & 56 & \\
\hline & & DG $(X, s$-é) & 2 & $(29)$ \\
\hline & & DG hors sub. & 1 & $(30)$ \\
\hline & & DG/topique cadre dans qu. partielle & 3 & (31) \\
\hline & & total $D G$ & 6 & \\
\hline & & DD dans exclamative & 2 & $(32)$ \\
\hline & & DD dans qu. totale & 1 & (33) \\
\hline & & DD dans qu. partielle & 3 & (34) \\
\hline & & total DD & 6 & \\
\hline & & clivée par $y$-a $X k$ & 4 & $(35)$ \\
\hline & & clivée par s-é & 1 & $(36)$ \\
\hline & $\mathrm{DG}+$ pr. tonique & $\mathrm{DG}+$ pr. tonique & 3 & $(37)$ \\
\hline & DG par ce & DG par $s$ & 1 & (38) \\
\hline & DG dans sub. & DG dans subordonnée & 1 & (39) \\
\hline & clivée par c'est & clivée par s-é & 2 & $(40)$ \\
\hline & VS dans qu. partielle & SV dans qu. partielle & 1 & $(41)$ \\
\hline pr. dém. & DD & DD & 1 & $(42)$ \\
\hline \multirow[t]{3}{*}{ gén. } & SV & RS avec $s$ & 1 & (43) \\
\hline & DG avec ça & DG/RS avec $s$ & 1 & $(44)$ \\
\hline & VS ds qu. part. & DD ds. qu. part. & 1 & $(45)$ \\
\hline
\end{tabular}

(26) é kãt la gamin al-é revnu 1-lãnmẽ, é bẽ 1 àn il-été pu dã sõ pré.

(st. no 70 : Mais quand la fillette revint le lendemain, l'âne n'était plus dans son pré.)

(27) tõ fré il-avé ti pà troué ou kat ãfã ?

(st. $\mathrm{n}^{\mathrm{o}} 75$ : Ton frère n'avait-il pas trois ou quatre enfant?)

(28) lavou dõ $\mathrm{k}$ mari a-tourv+ a-va trouvé du pẽ a 1 eur $\mathrm{k}$ il-é ?

(st. no 112 : Où Marie trouvera-t-elle du pain à cette heure-ci ?)

(29) bẽ la fiy $k$ é a kôté d lui, s-é ma niés.

(st. $\mathrm{n}^{\mathrm{o}} 11$ : Et la jeune fille à côté de lui est ma nièce.)

(30) noz ôt vouézẽ, sãblé $k$ il-avẽ désidé $d$ partir.

(st. $\mathrm{n}^{\circ} 238$ : Nos autres voisins semblaient avoir désidé de partir.)

(31) pi léz ôt, koué $k$ il-ã-pãsõ ?

(st. no $107:$ Qu'en pensent les autres ?) 
Tableau 5. Les sujets indéfinis de CR

\begin{tabular}{|c|c|c|c|c|}
\hline S du st. & réal. ds st. & réal. ds rép. & $\mathrm{nb}$ & ex. \\
\hline \multirow[t]{6}{*}{ DP indéf. } & SV & clivée par $y$-a $X$ ki/k & 17 & (46) \\
\hline & & clivée par $y$-a $X$ pour & 1 & (47) \\
\hline & & clivée par $y$-a $X d$ & 1 & (48) \\
\hline & & total clivées par y-a & 19 & \\
\hline & & impers. + VS & 2 & (49) \\
\hline & impers. + VS & impers. + VS & 1 & (47) \\
\hline
\end{tabular}

Tableau 6. Les sujets QU- de CR

\begin{tabular}{|c|c|c|c|c|}
\hline S du st. & réal. ds st. & réal. ds rép. & $\mathrm{nb}$ & ex. \\
\hline \multirow[t]{3}{*}{ pr. rel. qui } & antécédent déf. & $k i / k+V$ & 3 & (50) \\
\hline & antécédent indéf. & $k+\mathrm{V}$ & 5 & (51) \\
\hline & & total $\mathrm{ki} / \mathrm{k}+V$ & 7 & \\
\hline \multirow[t]{2}{*}{ mot QU } & qui & $q i(d \tilde{o}) \mathrm{k} / \mathrm{ki}$ & 4 & (52) \\
\hline & que & koué (dõ) $k$ & 2 & (53) \\
\hline \multirow[t]{3}{*}{ DP QU } & (le)quel & (1)qeul X ki & 2 & (54) \\
\hline & & qeul $X+\mathrm{RS}$ & 2 & (55) \\
\hline & & total $Q U$ & 10 & \\
\hline
\end{tabular}

(32) i-chãt ptét bẽ s merl!

(st. no 208 : Comme ce merle chante bien!)

(33) é-sõ chèr lê mézõ par isi ?

(st. no 219 : Les maisons sont-elles chères, par ici ?)

(34) qi dõ $\mathrm{k}$ vou-krouéyé $\mathrm{k}$ a-frékãt, sa fiy, a présã ?

(st. $\mathrm{n}^{\circ} 226$ : Qui croyez-vous que sa fille fréquente, à présent ?)

(35) $\quad y$-a marsèl $k$ a oubliyé $d$ yeu dir.

(st. $\mathrm{n}^{\circ} 122$ : Marcel a oublié de le leur dire.)

(36) s-é ma nié ki l-fra.

(st. $n^{\circ} 137$ : Ma nièce le fera.)

(37) sa fam, él, al-aré bẽ voulu résté, mé al-a pà pu.

(st. nº 235 : Sa femme, elle, elle aurait bien voulu resté, mais elle n'a pas pu.)

(38) tou nô vouézẽ s-é dê brav jã.

(st. $\mathrm{n}^{\circ} 325$ : Tous nos voisins, ce sont de braves gens.)

(39) lê gamẽ, fo $\mathrm{k}$ i-vién osi.

(st. $\mathrm{n}^{\circ} 271$ : Les enfants, il faut qu'ils viennent aussi.)

(40) s-é lé kouzẽ ki dvẽ fề sa.

(st. $\mathrm{n}^{\circ} 119$ : Ce sont les cousins qui devaient faire ça.)

(41) de qeul dõ kouleur ke lê volê sõ pẽ ?

(st. $\mathrm{n}^{\circ} 215$ : De quel couleur sont donc peints les volets ?)

(42) i-sra jamé séryeu, stél-la.

(st. no 88 : Sérieux, il ne le sera jamais, celui-là.)

(43) un ars s-é un outi pou kâsé lê mot.

(st. $n^{\circ} 286$ : Une herse est un outil pour casser les mottes.)

(44) la puré, sa devrá pà s-mãjé avék lê doué.

(st. nº 297 : La purée, ça ne devrait pas se manger avec les doigts.) 
(45) a koué dõ k sa-sér, s truk?

(st. no 285 : À quoi sert ce truc ?)

(46) ẽ jour, $y$-a un gamin ki s-é rtrouvé ã fas de lui.

(st. $\mathrm{n}^{\circ} 22$ : Un jour, une fillette se retrouva face à lui.)

(47) si tu-t-trouv avék el mouẽd dãjé, si i-t-ariv koué $k$ se soué, $j$-te-promé $k$ tou 1 tã y-ora qeukẽ pou t-êdé.

(st. no 61 : Si tu cours le moindre danger, s'il t'arrive quoi que ce soit, je te promets que, toujours, quelqu'un t'aidera.)

(48) j-kroué k y-ãn-a qeukẽ d pérdu.

(st. $\mathrm{n}^{\circ} 148$ : Je crois qu'il s'en est perdu quelques uns.)

(49) j-é sèrvi un asiété $d$ soup dê foué $k i$-vién qeukẽ.

(st. $\mathrm{n}^{\circ} 329$ : J'ai servi une assiette de soupe pour le cas où quelqu'un viendrait.)

(50) la gamin, ki s-lésé pà dékourajé, a-s-é pri a yi-kôzé.

(st. $\mathrm{n}^{\circ} 26$ : La fillette, que rien ne décourageait, lui adressa la parole.)

(51) $s$-é 1 istouèr $d$ un àn $k$ été kazimã jamé parti $d$ ché lui $+d$ sõ vilaj.

(st. no 14 : C'est l'histoire d'un âne qui n'avait presque jamais quitté son village.)

(52) si jamé ẽ jour tu-t-ã-và, qi dõ ki va alé ak toué?

(st. no 66 : Mais si tu décides de partir, qui penses-tu qui t'accompagnera?)

(53) koua dõ $k$ é tõbé?

(st. no 161 : Qu'est-ce qui est tombé ?)

(54) qeul dõ vouazin ki ira chérché mê nveu, dã s kà-la ?

(st. no 136 : Quelle voisine est-ce qui ira chercher mes neveux, alors?)

(55) qeul dõ pérson $k$ aré deu sou d jujot a-vouré alé viv la-bà ?

(st. $n^{\circ} 104$ : Quelle personne sensée accepterait d'aller y vivre ?) 